\title{
Motor Deficits Are Triggered by Reperfusion-Reoxygenation Injury as Diagnosed by MRI and by a Mechanism Involving Oxidants
}

\author{
Alexander Drobyshevsky, ${ }^{1}$ Kehuan Luo, ${ }^{1}$ Matthew Derrick, ${ }^{1}$ Lei Yu, ${ }^{1}$ Hongyan Du, ${ }^{2}$ P. V. Prasad, ${ }^{3}$ \\ Jeannette Vasquez-Vivar, ${ }^{4}$ Ines Batinic-Haberle, ${ }^{5}$ and Sidhartha Tan ${ }^{1}$ \\ ${ }^{1}$ Department of Pediatrics, ${ }^{2}$ Center on Outcomes, Research and Education, and ${ }^{3}$ Radiology, NorthShore University Healthcare Research Institute, Evanston, \\ Illinois 60201, ${ }^{4}$ Department of Biophysics, Medical College of Wisconsin, Milwaukee, Wisconsin 53226, and ${ }^{5}$ Department of Radiation Oncology, Duke \\ University Medical Center, Durham, North Carolina 27710
}

The early antecedents of cerebral palsy (CP) are unknown but are suspected to be due to hypoxia-ischemia (H-I). In our rabbit model of $\mathrm{CP}$, the MRI biomarker, apparent diffusion coefficient (ADC) on diffusion-weighted imaging, predicted which fetuses will develop postnatal hypertonia. Surviving H-I fetuses experience reperfusion-reoxygenation but a subpopulation manifested a continued decline of ADC during early reperfusion-reoxygenation, which possibly represented greater brain injury (RepRe0x). We hypothesized that oxidative stress in reperfusion-reoxygenation is a critical trigger for postnatal hypertonia. We investigated whether RepRe0x predicted postnatal neurobehavior, indicated oxidative stress, and whether targeting antioxidants at RepReOx ameliorated motor deficits, which included testing of a new superoxide dismutase mimic (MnTnHex-2-PyP). Rabbit dams, 79\% gestation (E25), were subjected to 40 min uterine ischemia. Fetal brain ADC was followed during H-I, immediate reperfusion-reoxygenation, and 4-72 h after H-I. Endpoints were postnatal neurological outcome at E32, ADC at end of H-I, ADC nadir during H-I and reperfusion-reoxygenation, and area under ADC curve during the first $20 \mathrm{~min}$ of reperfusion-reoxygenation. Antioxidants targeting RepReOx were administered before and/or after uterine ischemia. The new MRI-ADC biomarker for RepRe0x improved prediction of postnatal hypertonia. Greater superoxide production, mitochondrial injury, and oligodendroglial loss occurred in fetal brains exhibiting RepRe0x than in those without. The antioxidants, MnTnHex-2-PyP and Ascorbate and Trolox combination, significantly decreased postnatal motor deficits and extent of RepRe0x. The etiological link between early injury and later motor deficits can thus be investigated by MRI, and allows us to distinguish between critical oxidative stress that causes motor deficits and noncritical oxidative stress that does not.

\section{Introduction}

In adult stroke (Pan et al., 2007; Brouns and De Deyn, 2009) and myocardial ischemia (Yellon and Hausenloy, 2007; Ovize et al., 2010), significant injury is thought to occur during the reperfusion phase (Jung et al., 2010; Mazighi and Amarenco, 2011; Sanada et al., 2011). This has also been postulated in perinatal stroke (Gazzolo et al., 2009). However, most cases of perinatal injury involve global hypoxia and ischemia (H-I) and reperfusion rather than the focal injury of stroke. Reperfusion injury in fetal brain has been documented in cases of umbilical cord occlusion (Ikeda et al., 1999; Welin et al., 2005). Reperfusion injury in fetal brain has never been shown directly in cases of acute placental insufficiency such as abruptio placenta, possibly due to a lack of

Received Nov. 23, 2011; revised Feb. 1, 2012; accepted March 4, 2012

Author contributions: A.D., M.D., L.Y., P.P., J.V.-V., I.B.-H., and S.T. designed research; A.D., K.L., L.Y., J.V.-V., I.B.-H., and S.T. performed research; P.P., J.V.-V., I.B.-H., and S.T. contributed unpublished reagents/analytic tools; A.D., M.D., L.Y., H.D., J.V.-V., I.B.-H., and S.T. analyzed data; A.D., M.D., J.V.-V., I.B.-H., and S.T. wrote the paper.

Funding was provided by United Cerebral Palsy/Hearst foundation (A.D.), NIH NINDS Grants NS062367 (A.D.), NS063141, NS051402, NS043285 (S.T.), and NIH NICHD GrantHD057307 (M.D.).

Correspondence should be addressed to Alexander Drobyshevsky, Department of Pediatrics, NorthShore University Health Systems Research Institute, 2650 Ridge Avenue, Evanston, IL 60201. E-mail: oldrobys@gmail.com.

DOI:10.1523/JNEUROSCI.5986-11.2012

Copyright $\odot 2012$ the authors $\quad 0270-6474 / 12 / 325500-10 \$ 15.00 / 0$ suitable biomarkers. Since antepartum hypoxia-ischemia is a major cause of cerebral palsy (CP) (Gazzolo et al., 2009), we developed a model of CP involving global H-I and reperfusionreoxygenation (Tan et al., 2005).

Using fetal MRI in our model, we showed that the nadir of the apparent diffusion coefficient (ADC) in fetal brains predicted postnatal motor deficits in survivors (Drobyshevsky et al., 2007). In some cases, the ADC began to recover toward the baseline immediately after the end of H-I. In others, the ADC continued to decline further and reached nadir 5-15 min after the end of $\mathrm{H}-\mathrm{I}$. In these cases, the ADC recovery was slower and was associated with a worse postnatal neurobehavioral outcome. Thus, the ADC drop after end of H-I could represent biological changes during the reperfusion-reoxygenation phase, which most likely indicates injury to the brain. In this paper we define reperfusionreoxygenation injury as $\operatorname{RepReOx}$, and our primary hypothesis is that RepReOx is an initial trigger for later motor deficits. Studies on white matter injury following cord occlusion in fetal sheep (Welin et al., 2005) suggest a role of free radicals in reperfusion injury. Indirect evidence of oxidative stress has also been found in human brains (Haynes et al., 2005). Hence, our secondary hypothesis is that RepReOx is caused by oxidative stress. 
The systematic study of RepReOx represents advancement in elucidating early critical injury that results in adverse neurological outcome in CP. Previous studies did not have the benefit of an in vivo MRI biomarker to link early events to later motor deficits. To test our primary hypotheses, we first quantitatively assessed neurobehavioral outcome due to RepReOx. Then, we investigated whether oxidative stress only occurred in RepReOx. Finally, to confirm the critical role of oxidative stress, we tested whether subsequent motor deficits could be ameliorated by maternal administration of exogenous antioxidants targeting RepReOx, including a new potent manganese (Mn) porphyrinbased superoxide dismutase mimic.

\section{Materials and Methods}

The Institutional Animal Care and Use Committee of Evanston NorthShore University Healthcare approved all experimental procedures with animals.

Hypoxia-ischemia in the MRI magnet. The surgical procedure has been described in detail previously (Derrick et al., 2004). In vivo global H-I of fetuses was induced by sustained $40 \mathrm{~min}$ uterine ischemia at $25 \mathrm{~d}$ gestation $(79 \%$ term, E25) in timed pregnant New Zealand white rabbits (Myrtle's Rabbits). This procedure models acute placental insufficiency at a premature gestation. Briefly, dams were anesthetized with intravenous fentanyl $(75 \mu \mathrm{g} / \mathrm{kg} / \mathrm{h}$ ) and droperidol ( $3.75 \mathrm{mg} / \mathrm{kg} / \mathrm{h})$, followed by spinal anesthesia using $0.75 \%$ bupivicaine. A balloon catheter was introduced into the left femoral artery and advanced into the descending aorta to above the uterine and below the renal arteries. The catheterized animal was placed inside an MR scanner. Body core temperature was monitored with a rectal optic temperature probe (FOTS100, Biopac Systems) and maintained at $37 \pm 0.3^{\circ} \mathrm{C}$ with a water blanket wrapped around the dam's abdomen and connected to a temperature-controlled heating pump. After the dam was positioned in the magnet, the balloon was inflated for $40 \mathrm{~min}$ causing uterine ischemia and subsequent global fetal $\mathrm{H}-\mathrm{I}$. At the end of $\mathrm{H}-\mathrm{I}$, the balloon was deflated, resulting in uterine reperfusion and a reperfusion-reoxygenation period for fetal brains. After the imaging session, the catheter was removed, femoral artery repaired, and the dam was allowed to recover. MRI of the dam was repeated at 4, 24, and $72 \mathrm{~h}$ after H-I. Six days after H-I, at E31 (normal term E31.5), all fetuses were delivered by hysterotomy to ensure identification of uterine position of each fetus in relation to the previous MRI scans.

Outcome of newborn kits. Surviving kits at E32 underwent neurobehavioral assessment by two observers, masked to the treatment group assignment, to determine the presence of motor deficits and hypertonia as previously described (Derrick et al., 2004). The kits were categorized as follows: (1) Non-hypertonic kits with normal tone in all limbs. (2) Hypertonic kits with increased muscle tone. Hypertonia was defined as increased resistance to passive stretch in any limb. (3) Stillbirths, if fetuses were found dead on C-section at E31. (4) Miscarried fetuses, if found delivered in cage before C-section at E31.

Adverse outcome included stillbirths and presence of motor deficits. Since the reason of miscarriage was unknown and the exact time of death and presence of motor deficits could not be established in miscarried kits, those kits were excluded from final analysis.

Diffusion weighted MRI imaging during $H$-I. Dams were imaged serially in utero on a 3T Siemens Verio scanner using 8-channel knee coil. Highresolution single shot fast spin echo T2-weighted images (HASTE, TR/TE $1000 / 111 \mathrm{~ms}$, slice thickness $3 \mathrm{~mm}$, matrix $256 \times 157$, field of view $20 \mathrm{~cm}$ with $30-45$ axial slices covering all fetuses inside dam) were acquired in axial, coronal, and sagittal planes to identify sequential position of each fetus in uterine horns. Additional T2-weighted images (HASTE, TR/TE 1000/111 ms) were taken for anatomical reference in axial plane, with 25-32 axial slices covering all fetal brains. Slice thickness was $4 \mathrm{~mm}$, matrix $256 \times 157$, and field of view was $20 \mathrm{~cm}$. Single shot EPI diffusionweighted sequence with $b=0$ and $700 \mathrm{~s} / \mathrm{mm}^{2}$, TR/TE 6000/119 ms, 1 NEX, were acquired with the same geometry and resolution as the anatomical reference scan. Continuous acquisition of the diffusionweighted images was performed before, during H-I, and $20 \mathrm{~min}$ reperfusion with $40 \mathrm{~s}$ time resolution. Dams underwent three follow-up
MRI sessions at 4, 24, and $72 \mathrm{~h}$ after H-I to obtain anatomical and diffusion-weighted images at these time points.

MRI data analysis. ADC maps were calculated and image analysis was performed using in-house software, written in Matlab. The position of each fetus in each uterine horn and corresponding fetal brain was identified using orthogonal single shot anatomical datasets. Polygonal ROIs were placed on the whole fetal brains and transferred to the corresponding ADC maps. The position and shape of ROI placement was manually corrected for each time point to account for fetal head movement. We were able to track the position of each fetal brain within each uterine horn during the first and follow-up MRI sessions, and subsequently linked the MRI images to the delivered kits following hysterotomy. The evolution of an individual fetus's brain ADC response was followed from the initiation of $\mathrm{H}$-I through fetal to the postnatal period with MRI during acute $\mathrm{H}-\mathrm{I}$, immediate reperfusion, at 4, 24, and $72 \mathrm{~h}$ after, and associated with postnatal neurological outcome at E32. Outcome measures for brain tissue status on MRI were: $\mathrm{ADC}$ at the end of 40 min $\mathrm{H}-\mathrm{I}$, nadir of ADC during H-I, and reperfusion-reoxygenation, area under the $\mathrm{ADC}$ curves during reperfusion-reoxygenation phase $(0-20 \mathrm{~min}$ after H-I). Furthermore, if ADC declined further after the end of $\mathrm{H}-\mathrm{I}$ and the nadir occurred in the reperfusion-reoxygenation period, we diagnosed presence of RepReOx. We reasoned that the ADC change represented a biological change in the fetal brain, later shown to be associated with injury. A quantitative estimate of RepReOx was obtained from the area of the ADC curve during the first $20 \mathrm{~min}$ of reperfusion-reoxygenation period below the previously published threshold ADC value of $0.83 \times 10^{-3} \mathrm{~mm}^{2} / \mathrm{s}$.

Superoxide production in fetal brains. Superoxide production in fetal brains was determined in vivo by the conversion rate of a redox-sensitive probe hydroethidine (HE) into the specific product 2-hydroxyethidium $\left(2-\mathrm{OHE}^{+}\right)$. We defined superoxide production by the formation of $2-\mathrm{OHE}^{+}$detected in fetal brain homogenate. Since we expected the duration of free radical burst during reperfusion-reoxygenation to be short and access to fetuses in utero was limited, we chose to inject redoxsensitive probe HE intraperitoneally to fetuses before H-I. In this study, we used HPLC-electrochemical detection. HE was administered to fetuses intraperitoneally $300 \mu \mathrm{g} /$ fetus in a concentration of $1 \mathrm{mg} / \mathrm{ml}$ in 0.1 M PBS containing 20\% DMSO (Lewén et al., 2001) through a small laparotomy incision in E25 dams ( $n=2$ dams, 11 fetuses and 1 dam sham, 4 fetuses). The optimum dose and incubation time of HE to obtain signal was determined in optimization studies by varying HE dose and incubation times before $\mathrm{H}-\mathrm{I}$. Baseline fetal brain $\mathrm{ADC}$ after injection and before $\mathrm{H}$-I was not different from typical ADC $\left(1.25 \pm 0.15 \times 10^{-3} \mathrm{~mm}^{2} / \mathrm{s}\right)$ values without laparotomy. After closing the abdomen, the dams were transferred to a 3T MRI scanner for imaging. After H-I, fetal brains were extirpated and tissue frozen. Even after optimization of HE dose and time of the drug delivery, there was large variation between fetal brains in $2-\mathrm{OH}-\mathrm{E}^{+}$concentration between 0 and $10.7 \mathrm{pmol} / \mathrm{mg}$ protein in sham animals. To account for the difference between fetuses in HE accumulation in brains, and nonspecific conversion of $\mathrm{HE}$ to $\mathrm{E}+$, superoxide production was expressed as a ratio of $2-\mathrm{OH}-\mathrm{E}^{+}$to the sum of all detectable products, $2 \mathrm{OH}-\mathrm{E}^{+}, \mathrm{E}^{+}$, and $\mathrm{HE}$.

Mitochondrial function. Mitochondrial function was assessed by flow cytometry and staining for mitochondrial membrane potential. Fetal brains were extirpated after $20 \mathrm{~min}$ of reperfusion after H-I. A randomly chosen hemisphere was placed in ice-cold HBSS (Invitrogen ) $+10 \mathrm{~mm}$ HEPES, then the meninges were removed, and the cortex placed in $0.025 \%$ trypsin and incubated on a rotating shaker at $37^{\circ} \mathrm{C}$ for $45 \mathrm{~min}$. The brain suspension was spun at $300 \times g$ for $10 \mathrm{~min}$, the trypsin aspirated, and the cells washed with HBSS before limited trituration (30 times) in Neurobasal Media (Invitrogen ). The brain suspension was passed through a sterile $70 \mu \mathrm{m}$ filter to produce a single cell suspension. The cellular suspension was diluted to $1 \times 10^{6}$ cells per milliliter, incubated at $37^{\circ} \mathrm{C}$ for $15 \mathrm{~min}$ with cationic dye JC- 1 (5,5',6,6'-tetrachloro$1,1^{\prime}, 3,3^{\prime}$-tetraethylbenzimidazolocarbo cyanine iodide, Invitrogen) and assessed immediately on the flow cytometer (FACSCalibur, Becton Dickinson) (Derrick et al., 2001). We defined good mitochondrial function in a cell if the cell showed increased mitochondrial membrane potential. JC-1 is a lipophilic, cationic dye that can selectively enter into mitochondria and reversibly change color from green $(\sim 529 \mathrm{~nm})$ to red $(\sim 590$ $\mathrm{nm})$ as the membrane potential increases. Consequently, mitochondrial 
depolarization is indicated by a decrease in the red/green fluorescence intensity ratio. A separate aliquot of the brain cell suspension from each fetal brain was treated with valinomycin before incubation with JC-1 to validate the area on a scatter plot occupied by cells with low mitochondria membrane potential. The ionophore valinomycin effectively collapses mitochondria membrane potential and causes shift of probe fluorescence from red to green (Fig. 1) (Cossarizza and Salvioli, 2001). The ratio of number of cells with high to low mitochondrial membrane potential provided an index of mitochondrial function.

Oligodendroglial injury by flow cytometry. Fetal brains were harvested from E26 fetal rabbits, $24 \mathrm{~h}$ after H-I, and dissociated into single cell suspension using a previously described method (Mayer-Proschel, 2001). Cell numbers were counted with BD Aria II cell sorter (BD Biosciences) with CountBright absolute counting beads (Invitrogen). For each brain, an aliquot of $2 \times 10^{5}$ cells in $200 \mu \mathrm{l}$ was stained with primary anti-O4 antibody (Back et al., 2007), identifying oligodendrocytes progenitors, at the concentration of 1:1000 at room temperature for $30 \mathrm{~min}$, followed by two washes with PBS and 5\% BSA. FITC-conjugated secondary antibody was added at 1:1000 and incubated for 30 min followed by two washes. Cells were finally resuspended in 200 $\mu \mathrm{l}$ of PBS solution and run through BD Aria II for analysis. One microliter of propidium iodide (PI) solution $(1 \mathrm{mg} / \mathrm{ml})$ was added immediately before analysis to assess cell death. The cutoff gait was set using unstained cells with only $1 \%$ above the threshold of the fluorescent intensity. We defined live oligodendrocyte progenitors as cells that stained for $\mathrm{O} 4$ and healthy oligodendrocyte progenitors as cells that stained for $\mathrm{O} 4$ but were negative for PI.

Choice of Mn porphyrins from preliminary experiments with $H$-I at E22. Since our preliminary studies with HE revealed superoxide generation in fetal brains during $\mathrm{H}-\mathrm{I}$, we chose to test the neuroprotective effect of superoxide dismutase mimics that were designed to mimic the action of native superoxide dismutase in a much more efficient manner.

Following 40 min uterine ischemia in pregnant rabbits at E22, newborn rabbit kits manifest motor deficits on a neurobehavioral battery of tests at P1 (E32), very similar to cerebral palsy. We first tested administration of $60 \mathrm{ml}$ of 0.086 and $0.0086 \mathrm{~mm} \mathrm{Mn}(\mathrm{III})$ meso-tetrakis $(N-n$ hexylpyridinium-2-yl) porphyrin (MnTnHex-2-PyP) solution (0.12 and $1.2 \mathrm{mg} / \mathrm{kg}$ ) to rabbit dams or $1.05 \mathrm{~mm}$ MnTE-2-PyP, divided in two doses, $30 \mathrm{~min}$ before and $30 \mathrm{~min}$ after uterine ischemia, and compared with administration of $60 \mathrm{ml}$ of saline. Both MnTnHex-2-PyP groups had 14 normal, 2 mild, 3 severely affected P1 kits and 0 stillbirths compared with saline with 4 normal, 3 mild, 5 severe, and 6 stillbirths from 2 dams/group. Mn TE-2-PyP had 1 normal and 3 severe from one dam (Fig. 2). It was not surprising that MnTnHex-2-PyP had better neuroprotection than MnTE-2-PyP because it is more lipophilic, accumulates in mitochondria more readily, and is better able to cross the blood-brain barrier (Sheng et al., 2011).

Antioxidants with $H-I$ at E25. Having established feasibility, we chose MnTnHex-2-PyP infused intravenously (in an ear vein) to dam in the dose of $60 \mathrm{ml}$ of $0.085 \mathrm{~mm}$ at a rate of $60 \mathrm{ml} / \mathrm{h}$. In the first group, the entire dose was given after onset of H-I. In the second group, half the dose, $30 \mathrm{ml}$, was given before onset of $\mathrm{H}-\mathrm{I}$ and the other half after end of $\mathrm{H}-\mathrm{I}$. During uterine ischemia, it is likely that the drug would not reach the fetus. The antioxidant MnTnHex-2-PyP possesses exceptional ability to catalyze dismutation of superoxide and reduce peroxynitrite, thus decreasing levels of the reactive species.

A

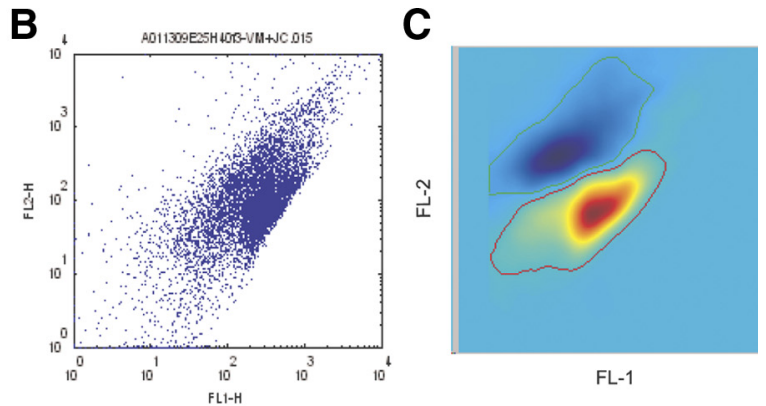

Figure 1. Mitochondrial function was assessed at $20 \mathrm{~min}$ after $\mathrm{H}-\mathrm{I}$. $\boldsymbol{A}$, Fetal brains were extirpated, dissociated into single cell suspension, incubated with potentiometric dye JC1, and measured on a flow cytometer. $\boldsymbol{B}$, Addition of ionophore valinomycin to a membrane potential. Proportion of cells in this region on original (top) scatter plot was calculated as an index of cells with

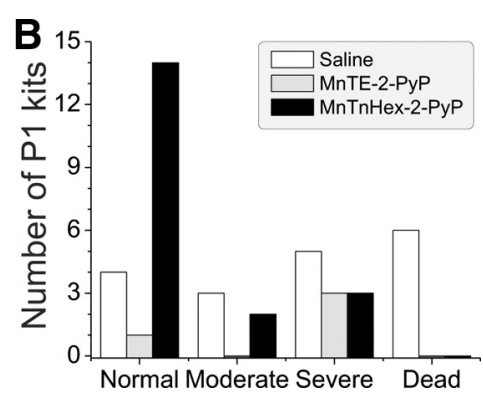
intravenously (in an ear vein) administered to the dam as a bolus of 100 $\mathrm{mg} / \mathrm{kg}$ Trolox (dissolved in $5 \mathrm{ml} / \mathrm{kg} 0.9 \%$ saline) and $1600 \mathrm{mg} / \mathrm{kg}$ ascorbic acid (dissolved in $5 \mathrm{ml} / \mathrm{kg} 0.9 \%$ saline). The bolus was infused after onset of and followed by a continuous infusion of $50 \mathrm{mg} / \mathrm{kg} / \mathrm{h}$ Trolox (concentration, $5 \mathrm{mg} / \mathrm{ml}$ ) and $60 \mathrm{mg} / \mathrm{kg} / \mathrm{h}$ ascorbic acid (concentration, 300 $\mathrm{mg} / \mathrm{ml}$ ) for $45 \mathrm{~min}$ (Tan S. et al., 1996) after the end of H-I. The control group got a saline infusion with volumes equivalent to the Ascorbate + Trolox experiment. As oxidative stress has been shown to occur relatively late after H-I, after the first $2 \mathrm{~h}$ (Miller et al., 2005; Welin et al., 2005) or after $4-6 \mathrm{~h}$ of reperfusion (Wang et al., 2009), we included a post-H-I treatment in all antioxidant groups. Since our primary end-point was hypertonia, measured $7 \mathrm{~d}$ after $\mathrm{H}-\mathrm{I}$, we did not want the later oxidative stress to be a factor in comparing hypertonia. The sham group underwent the same procedure as saline controls but instead of aortic occlusion for uterine ischemia, the balloon catheter was not inflated and there was no $\mathrm{H}-\mathrm{I}$ to fetuses. Number of dams and fetuses and stillbirths and miscarriages in each group are presented in Table 1.

Determination of MnTnHex-2-PyP tissue concentration by MRI. We found that MnTnHex-2-PyP has paramagnetic properties and used its T1 relaxation-shortening effect to determine concentration of the compound in maternal and fetal tissues ( $n=2$ dams and 8 fetuses). T1 relaxation maps were acquired during continuous intravenous infusion of MnTnHex-2-PyP, 30ml, $0.085 \mathrm{~mm}$, rate $60 \mathrm{ml} / \mathrm{h}$ to dams. 3D VFA sequences (available as a Siemens works-in-progress package) were used to estimate T1 relaxation maps (Deoni et al., 2005). The parameters for the $3 \mathrm{D}$ VFA sequence included TR/TE $=15 / 1.7 \mathrm{~ms}$, matrix size $=$ $192 \times 192 \times 20$, slice thickness $=5 \mathrm{~mm}$, bandwidth $=210 \mathrm{~Hz} /$ pixel. We used two flip angles, $4^{\circ}$ and $25^{\circ}$, acquisition, and inline T1 mapping with an acquisition time of $2 \mathrm{~min}$. For minimizing the effects of inherent $B_{1}$ inhomogeneities, $\mathrm{B}_{1}$ correction scheme was used. The method involves $\mathrm{B}_{1}$ mapping by comparing spin echo and corresponding stimulated echo 
Table 1. Number of dams and fetuses in treatment groups and neurological outcomes

\begin{tabular}{lclllcc}
\hline Groups & Dams & Fetuses at C-section & Non-hypertonic kits & Hypertonic kits & Stillbirths & Miscarriages in dams (fetuses) \\
\hline Sham & 2 & 16 & 16 & - & - & - \\
H-I saline & 12 & 96 & 28 & 38 & 30 & $1(12)$ \\
Antioxidants Ascorbate + Trolox, during reperfusion & 5 & 48 & 32 & 8 & 8 & $1(10)$ \\
Antioxidant MnTnHex-2-PyP, before H-I and during reperfusion & 7 & 51 & 36 & 10 & 11 & $2(18)$ \\
Antioxidant MnTnHex-2-PyP, during reperfusion & 5 & 41 & 8 & 14 & 20 & - \\
\hline
\end{tabular}

A

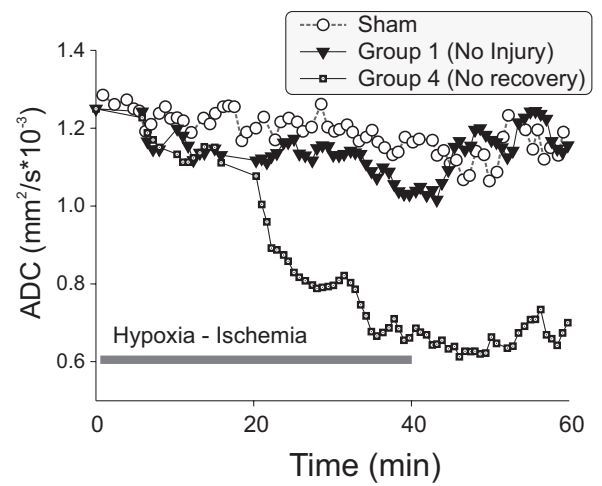

B

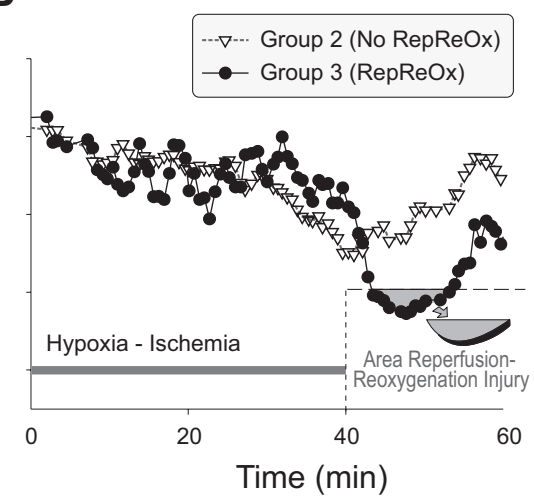

Figure 3. Time course of $A D C$ in fetal brains during $40 \mathrm{~min} \mathrm{H}-\mathrm{I}$ and reperfusion. $A$, Fetuses were classified based on the pattern of $A D C$ changes and especially during reperfusion. Group 1 showed no injury. Of the 7 fetuses in Group 1, none were dead or hypertonic. Group 4 had a deep fall in ADC that did not recover. Of the 9 fetuses 5 died and 4 were hypertonic. Sham fetuses were all normal. B, Showing the comparison with injury in $\mathrm{H}-\mathrm{I}$ alone (Group 2 without RepRe0x) and RepRe0x (Group 3). Of the 22 fetuses in Group 2, 1 was dead and 13 were hypertonic, compared with that in RepRe0x where of the 58 fetuses, 21 died and 29 were hypertonic. The amount of injury was estimated in RepRe0x by taking the area under the curve below an arbitrary line of $0.83 \times 10^{-3} \mathrm{~mm}^{2} / \mathrm{s}$ (from the threshold published before) and shown as a three-dimensional block.

(Akoka et al., 1993) and then applying a correction term for the regional flip angles. $\mathrm{B}_{1}$ mapping was performed at low spatial resolution and using a field of view (FOV) that was greater than that used for T1 mapping. The sequence parameters for the $2 \mathrm{D}$ multislice acquisition were $\mathrm{TR} / \mathrm{TE} / \mathrm{TM}=1368 / 14 / 14 \mathrm{~ms}$, matrix size $=64 \times 64, \mathrm{FOV}=250 \mathrm{~mm}$, slice thickness $=5 \mathrm{~mm}$.

T1 maps were acquired every $2 \mathrm{~min}$ for $80 \mathrm{~min}$ and 4 and $7 \mathrm{~h}$ thereafter. Time course of $\mathrm{T} 1$ change relative to the baseline before compound infusion was determined in ROIs placed on dam's vena cava, liver, placentas, and fetal liver and fetal brains. Relaxivity of the MnTnHex-2-PyP was determined in saline and in fresh tissue homogenates from dam's liver, blood, placentas, and fetal liver and brain in a separate session. MnTnHex-2-PyP was added to the tissue in known concentrations, obtained by serial dilution of $0.085 \mathrm{mM}$ of the compound from 1:1 to 1:256, and the samples were scanned within 30 min with the same sequence as for in vivo $\mathrm{T} 1$ determination. T1 relaxation times were obtained using standard inversion recovery spin-echo sequence with 12 inversion times from $23-2100 \mathrm{~ms}$. The difference between $\mathrm{T} 1$ values obtained by the two methods was minimal.

Relaxivity of MnTnHex-2-PyP measured in fresh tissue homogenates at room temperature $20^{\circ} \mathrm{C}$, in $\mathrm{mM}^{-1 *} \mathrm{sec}^{-1}$, was 17.26 in maternal blood, 12.09 in placenta, 17.28 in fetal liver, 10.28 in fetal brain, and 11.31 in saline. The relaxivity of the compound was relatively high, allowing determination of absolute concentration in living tissue with short acquisition and presence of motion. The method did not allow accurate determination of the compound concentration in big vessels with fast flow due to T2 shortening effect, so aortic and vena cava concentrations were not determined.

Determination of Ascorbate + Trolox accumulation in fetal tissues. Total antioxidant activity was determined using a fixed time point TRAP assay with modification of the method proposed by Rice-Evans and Miller (1994). Measurement of antioxidant activity is based on the inhibition by antioxidants of the absorbance of the radical cation of $\mathrm{ABTS}^{+}{ }^{+}$. The $\mathrm{ABTS}^{+}$radical is formed by the interaction of ABTS $(600 \mu \mathrm{M})$ with ferrylmyoglobin radical species, generated by the activation of metmyoglobin $(10 \mu \mathrm{M})$ with $\mathrm{H}_{2} \mathrm{O}_{2}$ reperfusion-reoxygenation.

(300 $\mu \mathrm{M})$. Timed absorbance readings were taken on a spectrophotometer every $7 \mathrm{~s}$ for $4 \mathrm{~min}$, at $25^{\circ} \mathrm{C}$. Samples were compared with a standard curve generated from similar absorbance readings obtained from known concentrations of Trolox in isotonic $5 \mathrm{~mm}$ PBS, $\mathrm{pH} 7.4$, at $25^{\circ} \mathrm{C}$. Total antioxidant activity in Trolox equivalents were determined in fetal blood, and brain samples were taken from different fetuses before occlusion (no antioxidants), and at 5, 10, and 15 min of reperfusion.

Microvascular blood flow with laser Doppler probe. Micro-vascular blood flow change in fetal brain was measured on a subset of dams $(n=3)$, by microprobe needle (probe 415 230) attached to a laser Doppler flow meter (PeriFlux System 5000, Perimed). After the dam was instrumented, a small $2 \mathrm{~cm}$ incision was made over the abdomen and the uterus was palpated for a fetal head. The microprobe was inserted $2 \mathrm{~mm}$ into fetal cortex through the uterine wall and retained in place manually until the end of the experiment. The abdominal incision was closed with slight pressure. The relative blood flow in arbitrary units was recorded before, during $\mathrm{H}-\mathrm{I}$, and during

Perfusion weighted imaging. Imaging was performed on a subset of dams $(n=4)$, twice for each dam, once before aortic balloon inflation (before $\mathrm{H}-\mathrm{I}$ ), and once $5 \mathrm{~min}$ after deflation (end of $\mathrm{H}-\mathrm{I}$ ), by quantifying image intensity changes after contrast injection. The perfusion sequence consisted of nine $6 \mathrm{~mm}$ slices with a $5 \mathrm{~mm}$ gap, acquired in the transverse plane of the dam's trunk using a fast T1-weighted SPGR sequence. Imaging parameters were as follows: TE $1.1 \mathrm{~ms}$, TR $3.3 \mathrm{~ms}$, flip angle 25, bandwidth $83.3,256 \times 64$ matrix, $18 \mathrm{~cm}$ FOV, $2 \mathrm{~s}$ per time point, 50 time points, no ECG triggering. The acquisition started 20 s before and continued during and after contrast intravenous injection of a bolus of $0.6 \mathrm{ml}$ containing $0.30 \mathrm{mmol} / \mathrm{kg}$ Magnevist (Gadopentetae dimeglumine, Berlex) through the ear vein, followed by saline flush $(3 \mathrm{ml})$. Bolus injection took $\sim 15 \mathrm{~s}$. A separate SSFSE scan was taken with the same slice geometry for in-plane anatomical reference to identify fetal brains. The slice package was placed in the center of abdomen, occupied by fetuses. Four to six individual fetal brains were identified in each rabbit dam for perfusion measurements. An index of fetal brain perfusion was estimated as slope of T1 signal enhancement (Li et al., 2000) when the contrast reached fetal brains. Relative changes in fetal brain perfusion were estimated as a ratio of the enhancement slopes analyzed for values obtained before and after H-I. Perfusion scans took $\sim 1.5$ min each and were performed between DWI series so relative perfusion changes and ADC time course could be registered for the same fetuses. These perfusion values provide an indirect and noninvasive method of measuring fetal brain perfusion as Magnevist is known to cross placental and blood brain barriers (Taillieu et al., 2006).

Statistical analysis. To account for within-litter data correlation, the differences between groups were tested using generalized estimating equations analysis, followed by post hoc multiple comparisons with Bonferroni correction using statistical package SAS 9.1. Data were presented as \pm SEM. Comparisons of hypertonia and stillbirth rate between group and controls was done using Fisher's exact test with Bonferroni correction for multiple comparisons. 
Table 2. Number of kits grouped by ADC pattern change during $\mathrm{H}-\mathrm{I}$ and reperfusion and neurological outcomes in the $\mathrm{H}-\mathrm{I}$ saline group

\begin{tabular}{|c|c|c|c|c|}
\hline ADC pattern & & & Dea & Total kits \\
\hline $\begin{array}{l}\text { Above top threshold during } \mathrm{H}-\mathrm{I} \text { and reperfusion } \\
\text { (Group 1) }\end{array}$ & 7 & 0 & 0 & 7 \\
\hline $\begin{array}{l}\text { No ADC drop during reperfusion (no RepRe0x } \\
\text { injury, Group 2) }\end{array}$ & 13 & 8 & 1 & 22 \\
\hline$A D C$ drop during reperfusion ( $\operatorname{Rep} R e 0 x$, Group 3) & 8 & 29 & 21 & 58 \\
\hline $\begin{array}{l}\text { Below bottom threshold during } \mathrm{H}-\mathrm{I} \text { (severe } \mathrm{H}-\mathrm{I} \\
\text { injury, Group 4) }\end{array}$ & 0 & 4 & 5 & 9 \\
\hline Total kits, rows & 28 & 41 & 27 & 96 \\
\hline
\end{tabular}

\section{Results}

\section{Diffusion weighted MRI indices of RepReOx injury}

Surviving fetuses were divided into four groups based on the ADC pattern and listed in increasing neurological severity (Fig. 3, Table 2), and compared with a sham surgery group: (1) ADC during $\mathrm{H}-\mathrm{I}$ and reperfusion that did not decline $<1.0 \times 10^{-3}$ $\mathrm{mm}^{2} / \mathrm{s}$, which previously predicted a normal neurodevelopment (Drobyshevsky et al., 2007) and was confirmed, as none of fetuses in this group had an adverse neurological outcome. Fetuses in this group were not included in further analysis of outcomes. (2) ADC at end of H-I that immediately recovered toward the baseline. This group constituted the comparison group for the next group with RepReOx and was indicated as the No RepReOx group. (3) ADC that declined further after the end of H-I. The biological changes underlying this pattern of ADC during reperfusion-reoxygenation period indicate RepReOx. (4) ADC that declined $<0.65 \times 10^{-3} \mathrm{~mm}^{2} / \mathrm{s}$ before the end of $\mathrm{H}-\mathrm{I}$ and did not recover during the first $20 \mathrm{~min}$ after end of $\mathrm{H}-\mathrm{I}$.

RepReOx occurred in $60.4 \%$ of all fetuses and in $65.7 \%$ of the fetuses with an ADC decline $<1.0 \times 10^{-3} \mathrm{~mm}^{2} / \mathrm{s}$ by the end of $\mathrm{H}-\mathrm{I}$ (i.e., excluding Group 1). RepReOx group accounted for $73.5 \%$ of all adverse outcomes (hypertonia or death), compared with $13.2 \%$ in fetuses without RepReOx (Fisher's exact test, $p<0.001$, Table 2). Miscarriages were not included in the count so the adverse outcome rate may be underestimated. The combination of RepReOx and ADC nadir improved the predictive value of the MRI biomarkers: absence of both biomarkers predicted no adverse outcome, presence of both biomarkers predicted $93.3 \%$, only ADC nadir, $86.2 \%$, and only RepReOx, 83\%, of adverse outcomes.

\section{Presence of RepReOx increases risk of hypertonia}

The rate of hypertonia, as determined by neurobehavioral testing (Derrick et al., 2004), in all surviving kits was 59.4\%. The rate of hypertonia was $78.4 \%$ in kits with RepReOx and $38.1 \%$ in kits without RepReOx (Fig. 4). Odds ratio of surviving kits with RepReOx to become hypertonic was 5.5 (confidence intervals 1.6-17.9, Fisher's exact test, $p<0.005$ ). Further examination of the MRI biomarker in Figure 2 showed that hypertonia rate in the RepReOx group proportionally increased with lower ADC at the end of $\mathrm{H}$-I period relative to fetuses in the No RepReOx group.

\section{Superoxide production is associated with $\mathrm{RepReOx}$}

Fetal brains that exhibited RepReOx had a significant increase in superoxide production (greater 2-hydroxyethidium production) compared with fetal brains without RepReOx, or fetal brains that were taken at the end of H-I (before deflating aortic balloon) or sham control brains (Fig. 5A). This is the first direct evidence of increased superoxide production in brain specifically in the immediate reperfusion-reoxygenation period.

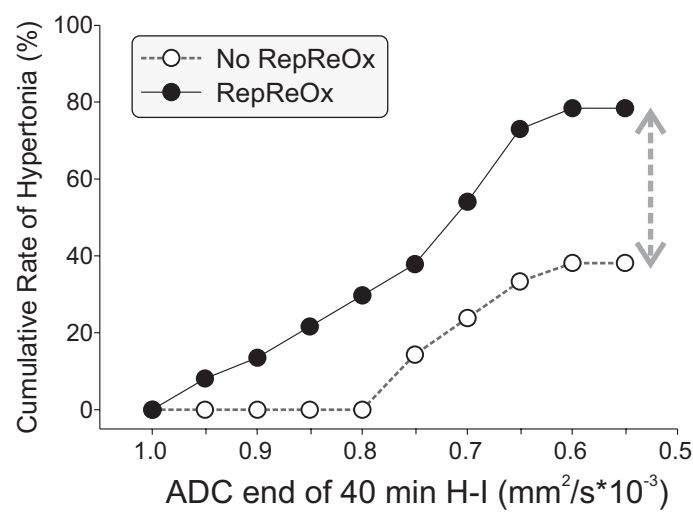

Figure 4. Comparison of all animals showing RepRe $0 x$ with a group with similar H-l injury as shown by the ADC at end of $40 \mathrm{~min} \mathrm{H-I}$. The presence of RepRe0x increases the chances of having hypertonia depending on the starting point at beginning of uterine reperfusion. The rate of hypertonia was 2.2 times higher in fetuses with RepRe0x that in those without RepRe0x (see arrow). Hypertonia only starts appearing in the No RepRe0x kits if they reach an $A D C<0.83 \times$ $10^{-3} \mathrm{~mm}^{2} / \mathrm{s}$ at end of $40 \mathrm{~min} \mathrm{H-I.} \mathrm{Note} \mathrm{that} \mathrm{the} \mathrm{abscissa} \mathrm{is} \mathrm{different} \mathrm{from} \mathrm{the} \mathrm{nadir} \mathrm{of} \mathrm{ADC,}$ which can occur after the end of $\mathrm{H}-\mathrm{I}$.

\section{Mitochondrial dysfunction in RepReOx}

The proportion of cells with good mitochondrial function was significantly less in the RepReOx group that in those in the No RepReOx group. This supports the reasoning that the ADC change showing RepReOx represents fetal brain injury (Fig. 5B). Mitochondrial dysfunction was greater in fetuses with the most severe H-I Injury (Group 4, 61.6 $\pm 1.9 \%$ cells with intact mitochondrial membrane potential). In view of previous studies linking white matter injury to fetal sheep H-I (Ikeda et al., 1999; Welin et al., 2005), we next investigated oligodendrocyte injury.

\section{Oligodendroglial Injury in $\mathrm{RepReOx}$}

The decrease in number of oligodendroglial precursors and immature oligodendrocytes $24 \mathrm{~h}$ after $\mathrm{H}$-I further supports the concept of brain injury in RepReOx (Fig. 5C).

\section{Targeting RepReOx by SOD mimic and antioxidants decrease} the rate of hypertonia

The SOD mimic, MnTnHex-2-PyP, when given before H-I decreased hypertonia rate to $21.7 \%$ (Fig. $6, p<0.05$ on Fisher's exact test with Bonferroni correction for multiple comparisons). Similarly administration of Ascorbate and Trolox as a combination $(\mathrm{A}+\mathrm{T})$ at reperfusion significantly decreased hypertonia to $20.0 \%$ in surviving kits compared with $59.4 \%$ in saline control group. In contrast, when $\mathrm{MnTnHex}-2-\mathrm{PyP}$ was administered at onset of reperfusion (after treatment), there was no protection.

\section{Quantification of protection by antioxidants by MRI biomarkers}

MnTnHex-2-PyP pretreatment decreased the area under the ADC cutoff during reperfusion-reoxygenation period by $30.0 \%$ and Ascorbate and Trolox decreased the area by $65.7 \%$ (Fig. 7), unlike MnTnHex-2-PyP post-treatment.

\section{Rate of antioxidant accumulation in fetuses}

In an attempt to explain the difference in neuroprotection between the antioxidants treatments, we subsequently measured the relative concentration of $\mathrm{MnTnHex}-2-\mathrm{PyP}$ in fetal tissues, including fetal brain, noninvasively by MRI, exploiting the T1shortening properties of the manganese compound. Concentration of the compound in maternal blood rose rapidly after 

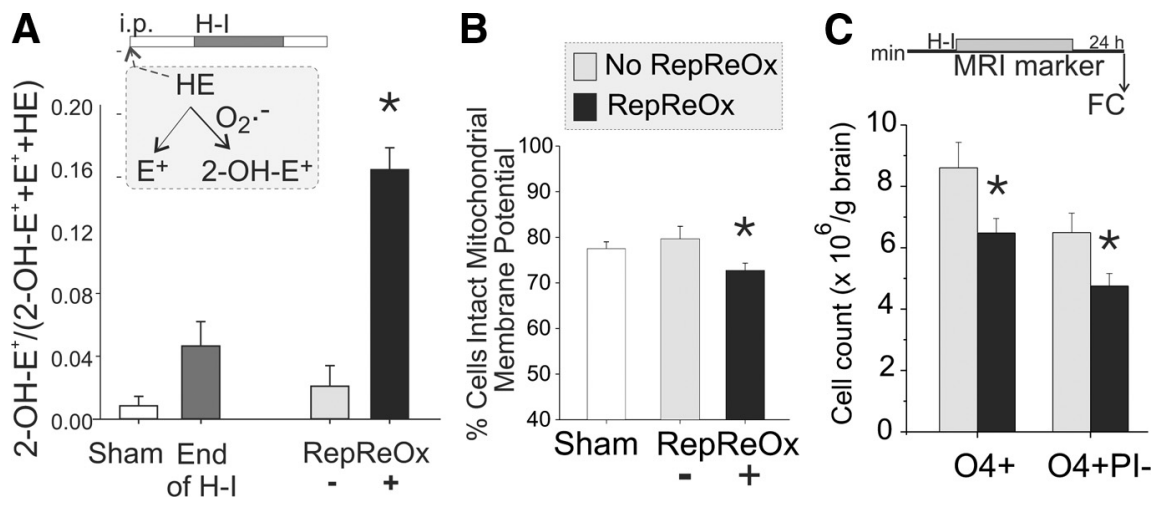

Figure 5. Superoxide formation and mitochondrial injury after globa1 fetal $\mathrm{H}-\mathrm{I}$ in $\mathrm{E} 25$ rabbit fetuses. $A$, Hydroethidine (HE) was administered to fetuses before H-I. Superoxide production in fetal brain was estimated by HPLC-ECD of 2-hydroxy-ethidium $\left(2-\mathrm{OH}-\mathrm{E}^{+}\right)$, a specific product of the reaction between $\mathrm{HE}$ and superoxide $\left(\mathrm{O}_{2}{ }^{-}\right)$. Ethidium $\left(\mathrm{E}^{+}\right)$is a nonspecific oxidation product of HE. Superoxide production was significantly higher in RepRe0x group $(+)$ compared with the one without $(-)$ or other groups. The No RepRe0x group (-) was not different from the sham and end of $\mathrm{H}-\mathrm{I}$ groups. Superoxide production is specific to the RepRe0x period. $\boldsymbol{B}$, Proportion of cells with intact mitochondrial membrane potential in fetal brain in the groups depicted in Figure 1. RepRe0x (Group 3) had significantly less cells with intact mitochondrial membrane potential compared with No RepRe0x or other groups. Group 4 that had decreased $A D C<0.65 \times 10^{-3} \mathrm{~mm}^{2} / \mathrm{s}$ and did not show recovery had the least number of cells with intact mitochondrial membrane potential. C, Number of viable oligodendrocyte progenitors were significantly smaller in RepRe0x group, assessed at $24 \mathrm{~h}$ after $\mathrm{H}-\mathrm{I}$. Brains were dissociated into single cell suspension and analyzed on flow cytometer using double staining identifying oligodendrocyte progenitors with 04 marker and dead or injured cells with propidium iodide. ${ }^{*} p<$ 0.05 ANOVA with post hoc comparisons.

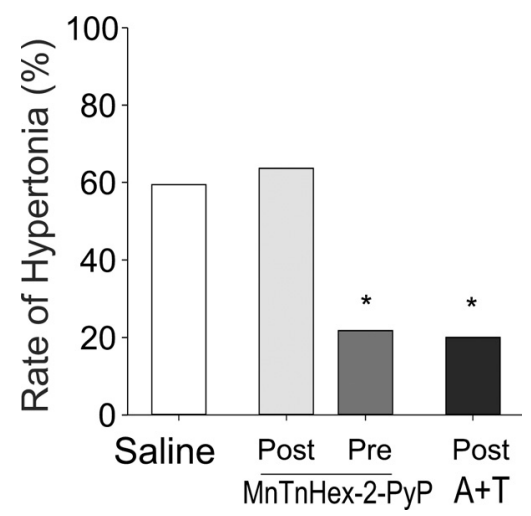

Figure 6. Decrease of hypertonia rate with antioxidant treatment targeted at RepRe0x. Antioxidants significantly decreased rate of hypertonia in surviving kits. When MnTnHex-2-PyP was administered after $\mathrm{H}-\mathrm{I}$ there was no change in hypertonia. Pretreatment of MnTnHex-2PyP or post-treatment with Ascorbate + Trolox $(A+T)$ significantly decreased hypertonia rate. ${ }^{*} p<0.05$ compared with $\mathrm{H}-\mathrm{I}$ saline group on Fisher's exact test.

beginning of infusion (data not shown), as indicated by T1 signal change. Concentration of the compound in placenta rose slowly (Fig. $8 \mathrm{~A}$ ) and appreciable accumulation of the compound in fetal brain $>0.002 \mathrm{~mm}$ was measured at 30-35 min time point. Concentration of the compound in fetal and maternal tissues decreased to almost to baseline levels within $3 \mathrm{~h}$ (Fig. $8 \mathrm{~B}$ ), except in maternal and fetal liver. Maternal liver was significantly enhanced on T1 weighted images at $4 \mathrm{~h}$ and even $24 \mathrm{~h}$ after infusion. The delay of the appearance of the compound in fetal brains from the beginning of intravenous maternal infusion can be attributed to partial clearance of the contrast during passage though maternal lungs until it reaches certain saturation level, and also placental and fetal blood-brain barriers. Despite lipophilic properties of MnTnHex-2-PyP, accumulation of the compound was not fast enough to prevent free radical damage immediately after reperfusion of ischemic fetuses. The delayed entry into fetal brain explains the lack of effect on reactive oxygen species and the difference between the protection between pre-administration and postadministration of MnTnHex-2-PyP.

Ascorbate and Trolox rapidly increased the total antioxidant activity in blood (two-fold at $5 \mathrm{~min}$ ) and in the fetal brain, an increase from the baseline was observed at $5 \mathrm{~min}$ and at $15 \mathrm{~min}$ of reperfusion using modified TRAP assay (Fig. 9).

Usefulness of MRI Biomarker at $24 \mathrm{~h}$

At $24 \mathrm{~h}$ after $\mathrm{H}-\mathrm{I}$ there was a significant decrease in ADC in fetuses that became hypertonic compared with nonhypertonic and sham fetuses. No differences were seen at 4 or $72 \mathrm{~h}$ after H-I. However, the ADC at $24 \mathrm{~h}$ was not as good a biomarker for prediction of hypertonia as the earlier biomarkers, because the absolute difference between hypertonic and non-hypertonic kits was small, $<0.02 \times$ $10^{-3} \mathrm{~mm}^{2} / \mathrm{s}$, and was not correlated with outcome if antioxidants were administered. ADC recovery was significantly higher for MnTnHex-2-PyP groups but not for Ascorbate+ Trolox compared with saline (Fig. 10).

Fetal brain perfusion in reperfusion-reoxygenation phase

To determine whether the ADC decline in the reperfusion phase in fetuses with RepReOx is truly related to reperfusion or is actually due to lack of reperfusion, we performed direct measurements of fetal cortical microvascular blood flow with laser Doppler probe (Fig. 11A). Microvascular perfusion started immediately after deflation of aortic balloon and end of uterine ischemia, rising to the levels of the preischemic state, and followed by a hyperperfusion period $<10$ min after the end of $\mathrm{H}-\mathrm{I}$.

We estimated an index of fetal brain perfusion as a slope of T1 signal enhancement in fetal brains after entry of contrast agent into fetal brain. The perfusion estimation was performed before $\mathrm{H}-\mathrm{I}$ and then again at $5 \mathrm{~min}$ after $\mathrm{H}-\mathrm{I}$. We did not observe any evidence of a lack of reperfusion at any ADC value around that time. There was no apparent relationship to the extent of ADC fall at 4-6 min after H-I (Fig. $11 B$ ).

\section{Discussion}

We have determined that certain fetuses undergo critical fetal brain injury following H-I. Using novel MRI biomarkers, RepReOx can be noninvasively identified in vivo, representing injury that is separate from injury that occurs during H-I. A key finding of this study is that the time of occurrence of the nadir of ADC (lowest ADC value) differed in those fetuses undergoing RepReOx (nadir during reperfusion-reoxygenation phase) and in those that did not (before the onset of reperfusionreoxygenation phase). The time of nadir thus determined our definition of whether the fetuses undergo RepReOx or not. All surviving fetuses undergo the same time of reperfusionreoxygenation after $\mathrm{H}-\mathrm{I}$ but may undergo some variable degree of injury during the reperfusion-reoxygenation phase. Only a subset of these fetuses will manifest injury to the extent that it can be detected by ADC as an added drop (defining RepReOx). Whether the injury in this definition of RepReOx is due to only to reperfusion-reoxygenation or due to accumulated injury from $\mathrm{H}-\mathrm{I}$ and reperfusion-reoxygenation is illus- 


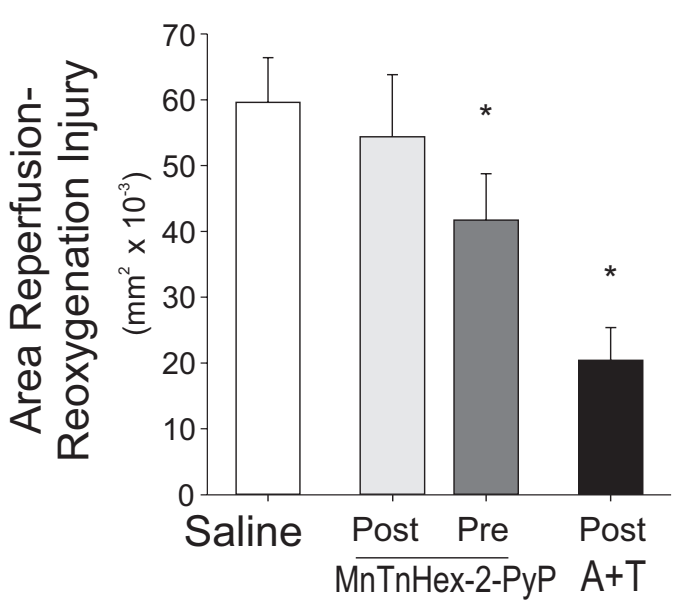

Figure 7. Pretreatment with MnTnHex-2-PyP and post-treatment with Ascorbate + Trolox $(A+T)$ decreased the area of reperfusion-reoxygenation injury on $A D C$ curve indicating less brain injury. ${ }^{*} p<0.05$ ANOVA with post hoc comparisons.
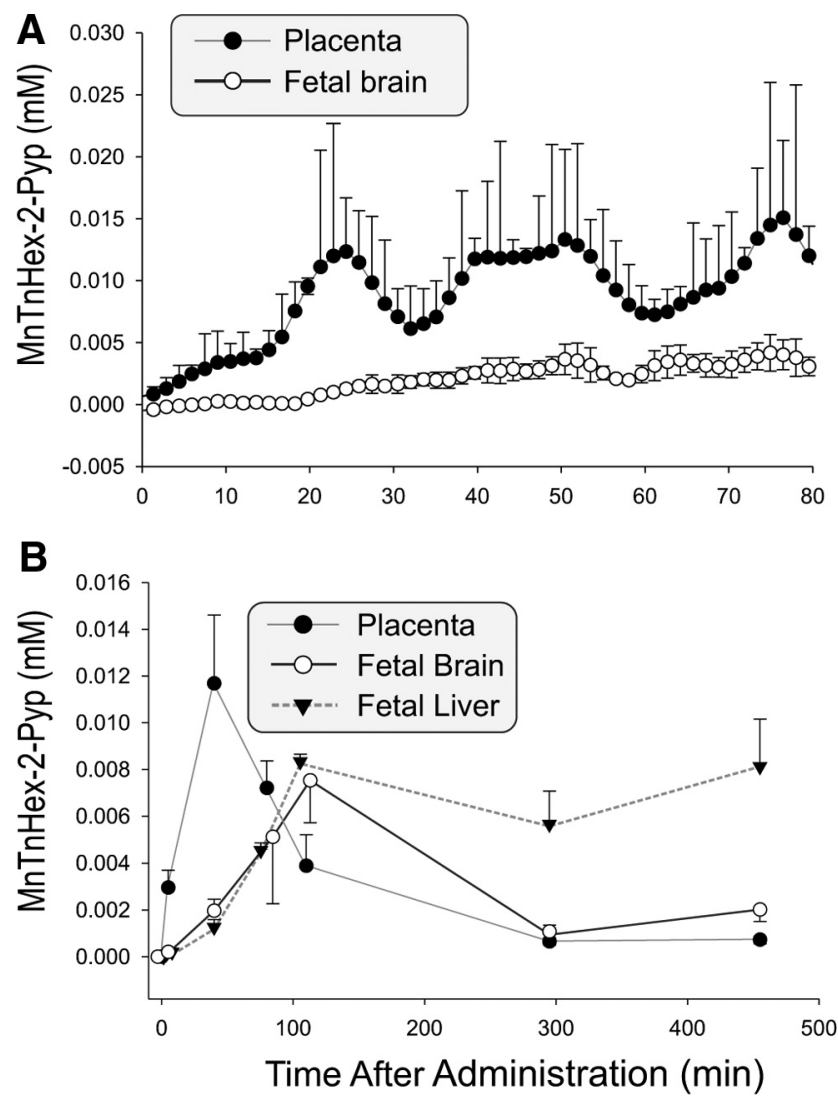

Figure 8. A, Time course of the MnTnHex-2-PyP accumulation in fetal tissues after maternal intravenous infusion of $30 \mathrm{ml} 0.085 \mathrm{~mm}$ of the compound within $20 \mathrm{~min}$ at E25. Absolute concentration of MnTnHex-2-PyP was estimated by change of $\mathrm{T} 1$ relaxation time multiplied by the compound T1 relaxivity, determine in tissue homogenates. Concentration of MnTnHex-2PyP reaches $0.002 \mathrm{~mm}$ in fetal brains $\sim 30-35$ min since the beginning of infusion. $\boldsymbol{B}$, Clearance of MnTnHex-2-PyP in fetal tissues determined on serial imaging of the same dam. Concentration of MnTnHex-2-PyP in placenta and fetal brain decreased to the baseline levels within $3 \mathrm{~h}$ but increased and remained at high levels in fetal liver.

trated in Figure 4. There is a small percentage of fetuses that manifest RepReOx at even high ADC levels at the end of H-I. These fetuses could be part of a group that predominantly suffers injury from events in the reperfusion-reoxygenation

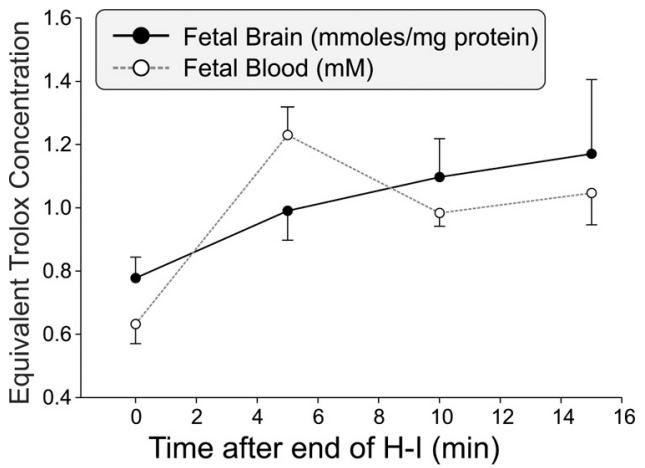

Figure 9. Dams were infused with $1600 \mathrm{mg} / \mathrm{kg}$ ascorbic acid and $100 \mathrm{mg} / \mathrm{kg}$ Trolox for duration of H-l after start of aortic occlusion and continued with $60 \mathrm{mg} / \mathrm{kg} / \mathrm{h}$ ascorbic acid and $50 \mathrm{mg} / \mathrm{kg} / \mathrm{h}$ Trolox after H-I. Fetal blood and brain samples were taken from different fetuses before occlusion (no antioxidants), and at 5, 10, and 15 min of reperfusion. Total tissue antioxidant capacity was measures by TRAP assay and expressed in Trolox equivalents. Brain antioxidant concentration was normalized by the tissue protein concentration. Concentration of antioxidants in maternal blood after Ascorbate + Trolox infusion was above upper limit of the assay calibration for fetal tissue $2 \mathrm{~mm}$.

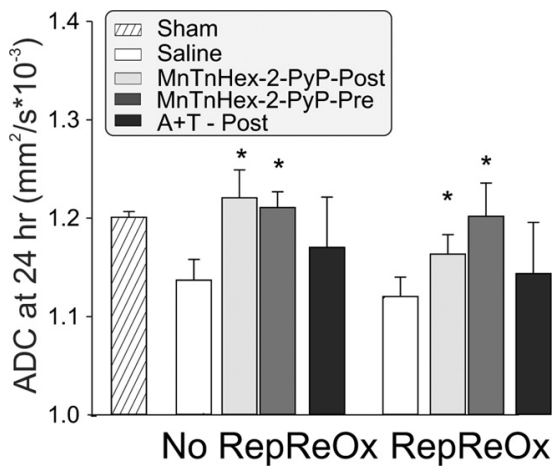

Figure 10. Surrogate marker of $A D C$ at $24 \mathrm{~h}$ after $\mathrm{H}-\mathrm{I}$ was not useful for predicting eventual outcome if antioxidants were administered. Improvement of brain function at $24 \mathrm{~h}$ was maximal in the MnTnHex-2-PyP groups including post-treatment. The post-MnTnHex-2-PyP findings confirm the importance of RepRe0x to development of hypertonia. Ascorbate + Trolox group showed no difference with saline at $24 \mathrm{~h}$, also indicating that short-lived protection of brain injury in RepRe0x is important for hypertonia. There was no significant difference in ADC between groups at $4 \mathrm{~h}$ after $\mathrm{H}-\mathrm{I} .{ }^{*} p<0.05$ difference from saline control group, ANOVA with post hoc comparisons.

phase. Most of the fetuses that undergo RepReOx will manifest cumulative injury from both injury in H-I and reperfusionreoxygenation periods.

The translational implication is that the fetus that is at a high risk for having subsequent adverse outcomes, including motor deficits, hence critical brain injury, can be identified. To the best of our knowledge, this is the first study to quantify the immediate brain ADC changes after $\mathrm{H}-\mathrm{I}$ and its effect on postnatal outcome. The current understanding of mechanisms of reperfusion brain injury originates from adult stroke models (Brouns and De Deyn, 2009). These may not necessarily be applicable to antenatal brain injury as mechanisms of cell death are quite distinctive (Ditelberg et al., 1996; McQuillen and Ferriero, 2004; Wang et al., 2009). Previous studies were focused on the delayed changes of ADC (>30 min) of focal H-I in adult (Neumann-Haefelin et al., 2000; Qiao et al., 2002) and postnatal animals (Nedelcu et al., 1999; Meng et al., 2005; Munkeby et al., 2008; Björkman et al., 2010), and thus on secondary energy failure.

Reperfusion after ischemia results in the restoration of oxygen and glucose and resumption of oxidative phosphorylation, and 

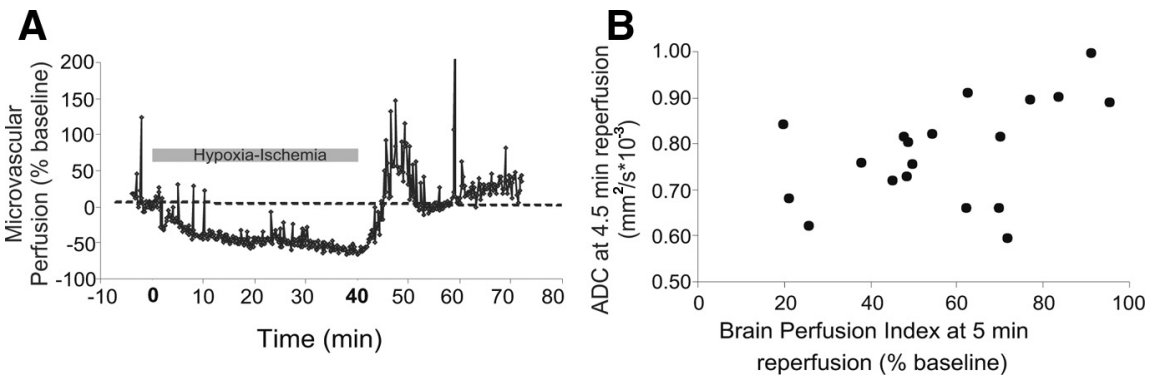

Figure 11. A, Micro-vascular blood flow change in fetal cortex measured using laser Doppler probe during $\mathrm{H}-\mathrm{I}$ and reperfusion. Cortical blood flow steadily dropped during $\mathrm{H}-\mathrm{I}$ phase accompanied by fetal bradicardia. With restoration of the blood flow to the uterus (arrow up), blood flow immediately began to recover and experience a period of hyper-perfusion 5-15 min later before normalization to the baseline. The experiment demonstrates that blood flow begins to recover immediately after cessation of $\mathrm{H}-\mathrm{I}$ and that we did not observe the phenomenon of "no-reflow" in any fetus. $\boldsymbol{B}$, Brain perfusion imaging with intravenous contrast infusion to dams before aortic balloon inflation and $5 \mathrm{~min}$ after aortic balloon deflation. Index of relative perfusion change was calculated as a ratio of slopes of signal enhancement before and after $\mathrm{H}-\mathrm{I}$. There was no significant relationship $\left(R^{2}=0.166, p=\right.$ 0.074 ) between recovery of cerebral perfusion at $5 \mathrm{~min}$ of reperfusion-reoxygenation and average $A D C$ values at $4-6 \mathrm{~min}$. Note that some degree of cerebral perfusion was always present at $5 \mathrm{~min}$ after $\mathrm{H}-\mathrm{I}$.

normalizes energy-demanding physiologic processes (Pulsinelli and Duffy, 1983). Paradoxically it may also result in deleterious biochemical processes in various organs, particularly the heart, and these constitute "reperfusion injury" (Jennings et al., 1960). Permanent blood flow occlusion results in smaller brain infarcts than in those with reversible occlusion (Aronowski et al., 1997; Pan et al., 2007; Yellon and Hausenloy, 2007). However, stroke constitutes a small fraction of perinatal $\mathrm{H}$-I brain injury (Wu et al., 2004), which is mostly due to global H-I. Hippocampal reperfusion injury has been demonstrated in global antenatal H-I from transient umbilical cord occlusion in fetal sheep (Fujii et al., 2003). This model usually results in delayed biochemical changes in reperfusion that constitute secondary energy failure (Tan WK et al., 1996; Dean et al., 2006).

\section{RepReOx and oxidative stress}

Oxidative stress is the most likely explanation for RepReOx, especially in the first $20 \mathrm{~min}$ after $\mathrm{H}-\mathrm{I}$. Fetuses exhibiting RepReOx have greater oxidative stress (Fig. 5A) compared with fetuses with no RepReOx in the same litter (hence, same insult). Targeted administration of antioxidants and MnTE-2-PyP attenuate postnatal hypertonia (Fig. 4). Importantly, changes in the first 20 min initiates an irreversible chain of events culminating in motor deficits. Later restorative systems for cellular homeostasis do not completely compensate for the injury marked by RepReOx. Following umbilical cord occlusion of fetal sheep, the major increase in free radical production is delayed and occurs after the first $2 \mathrm{~h}$ (Miller et al., 2005; Welin et al., 2005), although an early phase is also noticed in the first 60-90 min in one study (Miller et al., 2005). Even in umbilical cord occlusion in fetal sheep, only 4 of7 fetuses showed an increase in free radicals after 4-6 h of reperfusion; this increase was associated with greater brain injury (Wang et al., 2009). The new finding of the present study is that for the first time there is evidence of early oxidative stress immediately after global $\mathrm{H}-\mathrm{I}$ in fetuses, and this oxidative stress plays a critical etiological role in the generation of later motor deficits. The failure of post-MnTnHex-2-PyP to prevent postnatal motor deficits suggests that later oxidative stress may likely not play a major etiological role. Mitochondrial injury can lead to the failure of bioenergetics and inability of cells to restore ionic and water balance during reperfusion. MnTnHex-2-PyP accumulates in mitochondria where it can mimic mitochondrial matrix SOD (Batinic-Haberle et al., 2011). Mechanisms of mitochondrial and metabolic dysfunction include inhibition of the pyruvate dehydrogenase complex by free radicals, loss of mitochondrial $\mathrm{NAD}(\mathrm{H})$ through the permeability transition pore, consumption of $\mathrm{NAD}^{+}$by poly-ADP ribose polymerase 1 , and release of cytochrome $c$ (Robertson et al., 2009). Early mitochondrial injury as evidenced by changes in mitochondrial membrane potential was also observed in RepReOx (Fig. 5B). The exact biophysical nature of ADC changes in $\mathrm{RepReOx}$ is still unclear. ADC changes are linked to the status of bioenergetics of cells and ability to maintain water and ion homeostasis (van der Toorn et al., 1996; van Pul et al., 2005). However, mitochondrial dysfunction was the greatest in the severe $\mathrm{H}-\mathrm{I}$ injury group (Group 4), indicating that if anything, the smaller mitochondrial dysfunction in RepReOx was associated with oxidative stress rather than from energy failure alone.

In fetal sheep umbilical cord occlusion, there is an initial cerebral hyper-perfusion that peaks at 6 min after end of occlusion followed by hypo-perfusion that reaches nadir at $90 \mathrm{~min}$ (Dean et al., 2006). The late hypo-perfusion was not observed with shorter occlusion in later gestation sheep (Fujii et al., 2003). If there was delayed hypo-perfusion in our rabbit fetuses, it should have resulted in a delayed ADC fall, but ADC at $4 \mathrm{~h}$ was not different. The point is that RepReOx is not likely from either hypo-perfusion or the "no-reflow" phenomenon observed after stroke (del Zoppo and Mabuchi, 2003), because the timing is incongruent (Davis et al., 1994).

\section{ADC is a marker of added injury}

In our previous study, ADC nadir was highly predictive of adverse postnatal neurological outcome (Drobyshevsky et al., 2007). By adding the ADC decline in reperfusion, the positive predictive value of early ADC markers for adverse postnatal neurological outcome improves to $93.3 \%$ from $83.3 \%$. Remarkably, positive predictive value for normal postnatal neurological outcome with the absence of both ADC biomarkers was 100\%. As a marker, diffusion-weighted imaging complements existing MRI and electron paramagnetic resonance (EPR) for redox-imaging, and does not require custom-built variable field scanners (Yamato et al., 2009) or injection of blood-brain barrier-permeable redox-sensitive probes for EPR (Yokoyama et al., 2004) or MRI (Hyodo et al., 2008). The noninvasive marker with high temporal and spatial resolution enables the investigation of early oxidative stress without the need to kill animals and thus lose the ability to associate delayed neurological sequelae. This allows a direct link to be made from early events to multiple pathways, including cell death continuum and inflammation that result in neonatal brain damage. MRI is most useful for investigating not only the early triggers but the critical delayed cellular events as shown in Figure $5 C$. A detailed study of gray and white matter injury is a future endeavor outside the scope of this manuscript. It is possible that, like the case of oxidative stress, not all delayed events result in motor deficits.

Postnatal hypertonia increased significantly as the severity of initial H-I injury increased, as indicated by ADC at the end of H-I (Fig. 4). Added injury, measured as increased postnatal hypertonia rate with RepReOx given the same ADC at the end of $\mathrm{H}-\mathrm{I}$, was 
also small when severity of insult during H-I, determined by ADC at end of $\mathrm{H}$-I period, was small (Fig. 4), and gradually increased with increased severity of $\mathrm{H}$-I insult, suggesting that $\mathrm{H}$-I injury is necessary for RepReOx to occur. The extent of reperfusion injury depends on the severity of initial insult during myocardial ischemia (Ovize et al., 2010). In a piglet model of global H-I, the significant correlation between reduction in cerebral perfusion and cerebral ADC during $\mathrm{H}-\mathrm{I}$ was lost during the reperfusion period (Munkeby et al., 2004). It implies that ADC changes and the corresponding cellular damage during reperfusion is not a yes/no condition but may be a function of degree and duration of the initial hypo-perfusion in uterine ischemia. Fast recovery of $\mathrm{ADC}$ indicates that the initial injury is probably reversible, but delayed ADC recovery is associated with adverse outcome (Miyasaka et al., 2000).

\section{Fast delivery of antioxidants to brain is necessary for protection}

The similar decrease in the postnatal hypertonia rate after pretreatment with MnTnHex-2-PyP and post-treatment with Ascorbate+Trolox (Fig. 6) suggests that the neuroprotection depends on presence of antioxidants in the fetal brain when free radical production is highest, and less on the antioxidant efficiency of the therapy. The lack of effect with post-treatment with MnTnHex-2-PyP suggests that delayed oxidant production was not a major etiological factor in motor deficits due to antenatal H-I. The development of the future antioxidant agents and therapeutic strategies should target rapid delivery to the perinatal brain, and efficacy can be assessed with MRI biomarkers.

Differences in accumulation and retention of antioxidants in fetal brains may explain the delayed recovery of ADC at $24 \mathrm{~h}$ of MnTnHex-2-PyP post-treatment compared with the insignificant effect of Ascorbate+Trolox (Fig. 10). The delayed effect of MnTnHex-2-PyP raises the possibility of delayed oxidative injury playing a role in the development of later brain injury.

\section{Conclusions}

In conclusion, we provide evidence that the diagnosis of brain injury, occurring early in the reperfusion-reoxygenation period, can be made using MRI with a further drop in ADC, indicating RepReOx. The brain injury in fetuses with RepReOx adversely contributes to the eventual hypertonia. ADC change is influenced by both oxidative stress and mitochondrial dysfunction. Antioxidants are viable candidates for neuroprotection when given either before or after the onset of H-I, but adequate levels need to obtained early in reperfusion, otherwise the protective effects are lost. The novel noninvasive RepReOx index, indicative of oxidative and mitochondrial injury, can guide development of drugs targeting critical period of early reperfusion-reoxygenation after H-I.

\section{References}

Akoka S, Franconi F, Seguin F, Le Pape A (1993) Radiofrequency map of an NMR coil by imaging. Magn Reson Imaging 11:437-441.

Aronowski J, Strong R, Grotta JC (1997) Reperfusion injury: demonstration of brain damage produced by reperfusion after transient focal ischemia in rats. J Cereb Blood Flow Metab 17:1048-1056.

Back SA, Craig A, Kayton RJ, Luo NL, Meshul CK, Allcock N, Fern R (2007) Hypoxia-ischemia preferentially triggers glutamate depletion from oligodendroglia and axons in perinatal cerebral white matter. J Cereb Blood Flow Metab 27:334-347.

Batinic-Haberle I, Rajic Z, Tovmasyan A, Reboucas JS, Ye X, Leong KW, Dewhirst MW, Vujaskovic Z, Benov L, Spasojevic I (2011) Diverse functions of cationic $\mathrm{Mn}(\mathrm{III}) \mathrm{N}$ - substituted pyridylporphyrins, recognized as SOD mimics. Free Radic Biol Med 51:1035-1053.
Björkman ST, Miller SM, Rose SE, Burke C, Colditz PB (2010) Seizures are associated with brain injury severity in a neonatal model of hypoxiaischemia. Neuroscience 166:157-167.

Brouns R, De Deyn PP (2009) The complexity of neurobiological processes in acute ischemic stroke. Clin Neurol Neurosurg 111:483-495.

Cossarizza A, Salvioli S (2001) Flow cytometric analysis of mitochondrial membrane potential using JC-1. Curr Protoc Cytom Chapter 9:Unit 9. 14.

Davis D, Ulatowski J, Eleff S, Izuta M, Mori S, Shungu D, van Zijl PC (1994) Rapid monitoring of changes in water diffusion coefficients during reversible ischemia in cat and rat brain. Magn Reson Med 31:454-460.

Dean JM, Gunn AJ, Wassink G, Bennet L (2006) Transient NMDA receptor-mediated hypoperfusion following umbilical cord occlusion in preterm fetal sheep. Exp Physiol 91:423-433.

del Zoppo GJ, Mabuchi T (2003) Cerebral microvessel responses to focal ischemia. J Cereb Blood Flow Metab 23:879-894.

Deoni SC, Peters TM, Rutt BK (2005) High-resolution T1 and T2 mapping of the brain in a clinically acceptable time with DESPOT1 and DESPOT2. Magn Reson Med 53:237-241.

Derrick M, He J, Brady E, Tan S (2001) The in vitro fate of rabbit fetal brain cells after acute in vivo hypoxia. J Neurosci 21:RC138.

Derrick M, Luo NL, Bregman JC, Jilling T, Ji X, Fisher K, Gladson CL, Beardsley DJ, Murdoch G, Back SA, Tan S (2004) Preterm fetal hypoxiaischemia causes hypertonia and motor deficits in the neonatal rabbit: a model for human cerebral palsy? J Neurosci 24:24-34.

Ditelberg JS, Sheldon RA, Epstein CJ, Ferriero DM (1996) Brain injury after perinatal hypoxia-ischemia is exacerbated in copper/zinc superoxide dismutase transgenic mice. Pediatr Res 39:204-208.

Drobyshevsky A, Derrick M, Prasad PV, Ji X, Englof I, Tan S (2007) Fetal brain magnetic resonance imaging response acutely to hypoxia-ischemia predicts postnatal outcome. Ann Neurol 61:307-314.

Fujii EY, Takahashi N, Kodama Y, Roman C, Ferriero DM, Parer JT (2003) Hemodynamic changes during complete umbilical cord occlusion in fetal sheep related to hippocampal neuronal damage. Am J Obstet Gynecol 188:413-418.

Gazzolo D, Abella R, Marinoni E, Di Iorio R, Li Volti G, Galvano F, Pongiglione G, Frigiola A, Bertino E, Florio P (2009) Circulating biochemical markers of brain damage in infants complicated by ischemia reperfusion injury. Cardiovasc Hematol Agents Med Chem 7:108-126.

Haynes RL, Baud O, Li J, Kinney HC, Volpe JJ, Folkerth DR (2005) Oxidative and nitrative injury in periventricular leukomalacia: a review. Brain Pathol 15:225-233.

Hyodo F, Chuang KH, Goloshevsky AG, Sulima A, Griffiths GL, Mitchell JB, Koretsky AP, Krishna MC (2008) Brain redox imaging using bloodbrainbarrier-permeable nitroxide MRI contrast agent. J Cereb Blood Flow Metab 28:1165-1174.

Ikeda T, Choi BH, Yee S, Murata Y, Quilligan EJ (1999) Oxidative stress, brain white matter damage and intrauterine asphyxia in fetal lambs. Int $J$ Dev Neurosci 17:1-14.

Jennings RB, Sommers HM, Smyth GA, Flack HA, Linn H (1960) Myocardial necrosis induced by temporary occlusion of a coronary artery in the dog. Arch Pathol 70:68-78.

Jung JE, Kim GS, Chen H, Maier CM, Narasimhan P, Song YS, Niizuma K, Katsu M, Okami N, Yoshioka H, Sakata H, Goeders CE, Chan PH (2010) Reperfusion and neurovascular dysfunction in stroke: from basic mechanisms to potential strategies for neuroprotection. Mol Neurobiol 41:172-179.

Lewén A, Sugawara T, Gasche Y, Fujimura M, Chan PH (2001) Oxidative cellular damage and the reduction of APE/Ref- 1 expression after experimental traumatic brain injury. Neurobiol Dis 8:380-390.

Li W, Brophy DP, Chen Q, Edelman RR, Prasad PV (2000) Semiquantitative assessment of uterine perfusion using first pass dynamic contrastenhanced MR imaging for patients treated with uterine fibroid embolization. J Magn Reson Imaging 12:1004-1008.

Mayer-Proschel M (2001) Isolation and generation of oligodendrocytes by immunopanning. Curr Protoc Neurosci Chapter 3:Unit 3. 13.

Mazighi M, Amarenco P (2011) Reperfusion therapy in acute cerebrovascular syndrome. Curr Opin Neurol 24:59-62.

McQuillen PS, Ferriero DM (2004) Selective vulnerability in the developing central nervous system. Pediatr Neurol 30:227-235.

Meng S, Qiao M, Foniok T, Tuor UI (2005) White matter damage precedes that in gray matter despite similar magnetic resonance imaging changes 
following cerebral hypoxia-ischemia in neonatal rats. Exp Brain Res 166:56-60.

Miller SL, Yan EB, Castillo-Meléndez M, Jenkin G, Walker DW (2005) Melatonin provides neuroprotection in the late-gestation fetal sheep brain in response to umbilical cord occlusion. Dev Neurosci 27:200-210.

Miyasaka N, Nagaoka T, Kuroiwa T, Akimoto H, Haku T, Kubota T, Aso T (2000) Histopathologic correlates of temporal diffusion changes in a rat model of cerebral hypoxia/ischemia. AJNR Am J Neuroradiol 21:60-66.

Munkeby BH, Lyng K, Frøen JF, Winther-Larssen EH, Rosland JH, Smith HJ, Saugstad OD, Bjørnerud A (2004) Morphological and hemodynamic magnetic resonance assessment of early neonatal brain injury in a piglet model. J Magn Reson Imaging 20:8-15.

Munkeby BH, De Lange C, Emblem KE, Bjørnerud A, Kro GA, Andresen J, Winther-Larssen EH, Løberg EM, Hald JK (2008) A piglet model for detection of hypoxic-ischemic brain injury with magnetic resonance imaging. Acta Radiol 49:1049-1057.

Nedelcu J, Klein MA, Aguzzi A, Boesiger P, Martin E (1999) Biphasic edema after hypoxic-ischemic brain injury in neonatal rats reflects early neuronal and late glial damage. Pediatr Res 46:297-304.

Neumann-Haefelin T, Kastrup A, de Crespigny A, Yenari MA, Ringer T, Sun GH, Moseley ME (2000) Serial MRI after transient focal cerebral ischemia in rats: dynamics of tissue injury, blood-brainbarrier damage, and edema formation. Stroke 31:1965-1972; discussion 1972-1973.

Ovize M, Baxter GF, Di Lisa F, Ferdinandy P, Garcia-Dorado D, Hausenloy DJ, Heusch G, Vinten-Johansen J, Yellon DM, Schulz R (2010) Postconditioning and protection from reperfusion injury: where do we stand? Position paper from the Working Group of Cellular Biology of the Heart of the European Society of Cardiology. Cardiovasc Res 87:406-423.

Pan J, Konstas AA, Bateman B, Ortolano GA, Pile-Spellman J (2007) Reperfusion injury following cerebral ischemia: pathophysiology, MR imaging, and potential therapies. Neuroradiology 49:93-102.

Pulsinelli WA, Duffy TE (1983) Regional energy balance in rat brain after transient forebrain ischemia. J Neurochem 40:1500-1503.

Qiao M, Malisza KL, Del Bigio MR, Tuor UI (2002) Transient hypoxia-ischemia in rats: changes in diffusion-sensitive MR imaging findings, extracellular space, and $\mathrm{Na}+-\mathrm{K}+$-adenosine triphosphatase and cytochrome oxidase activity. Radiology 223:65-75.

Rice-Evans C, Miller NJ (1994) Total antioxidant status in plasma and body fluids. Methods Enzymol 234:279-293.

Robertson CL, Scafidi S, McKenna MC, Fiskum G (2009) Mitochondrial mechanisms of cell death and neuroprotection in pediatric ischemic and traumatic brain injury. Exp Neurol 218:371-380.

Sanada S, Komuro I, Kitakaze M (2011) Pathophysiology of myocardial reperfusion injury: preconditioning, postconditioning, and translational aspects of protective measures. Am J Physiol Heart Circ Physiol 301:H1723-1741.

Sheng H, Spasojevic I, Tse HM, Jung JY, Hong J, Zhang Z, Piganelli JD,
Batinic-Haberle I, Warner DS (2011) Neuroprotective efficacy from a lipophilic redox-modulating $\mathrm{Mn}$ (III) $\mathrm{N}$-hexylpyridylporphyrin, MnTnHex-2-PyP: rodent models of ischemic stroke and subarachnoid hemorrhage. J Pharmacol Exp Ther 338:906-916.

Taillieu F, Salomon LJ, Siauve N, Clément O, Faye N, Balvay D, Vayssettes C, Frija G, Ville Y, Cuenod CA (2006) Placental perfusion and permeability: simultaneous assessment with dual-echo contrast-enhanced MR imaging in mice. Radiology 241:737-745.

Tan S, Liu YY, Nielsen VG, Skinner K, Kirk KA, Baldwin ST, Parks DA (1996) Maternal infusion of antioxidants (Trolox and ascorbic acid) protects the fetal heart in rabbit fetal hypoxia. Pediatr Res 39:499-503.

Tan S, Drobyshevsky A, Jilling T, Ji X, Ullman LM, Englof I, Derrick M (2005) Model of cerebral palsy in the perinatal rabbit. J Child Neurol 20:972-979.

Tan WK, Williams CE, During MJ, Mallard CE, Gunning MI, Gunn AJ, Gluckman PD (1996) Accumulation of cytotoxins during the development of seizures and edema after hypoxic-ischemic injury in late gestation fetal sheep. Pediatr Res 39:791-797.

van der Toorn A, Syková E, Dijkhuizen RM, Vorísek I, Vargová L, Skobisová E, van Lookeren Campagne M, Reese T, Nicolay K (1996) Dynamic changes in water ADC, energy metabolism, extracellular space volume, and tortuosity in neonatal rat brain during global ischemia. Magn Reson Med 36:52-60.

van Pul C, Jennekens W, Nicolay K, Kopinga K, Wijn PF (2005) Ischemiainduced ADC changes are larger than osmotically-induced ADC changes in a neonatal rat hippocampus model. Magn Reson Med 53:348-355.

Wang X, Carlsson Y, Basso E, Zhu C, Rousset CI, Rasola A, Johansson BR, Blomgren K, Mallard C, Bernardi P, Forte MA, Hagberg H (2009) Developmental shift of cyclophilin D contribution to hypoxic-ischemic brain injury. J Neurosci 29:2588-2596.

Welin AK, Sandberg M, Lindblom A, Arvidsson P, Nilsson UA, Kjellmer I, Mallard C (2005) White matter injury following prolonged free radical formation in the 0.65 gestation fetal sheep brain. Pediatr Res 58:100-105.

Wu YW, March WM, Croen LA, Grether JK, Escobar GJ, Newman TB (2004) Perinatal stroke in children with motor impairment: a population-based study. Pediatrics 114:612-619.

Yamato M, Shiba T, Yamada K, Watanabe T, Utsumi H (2009) Noninvasive assessment of the brain redox status after transient middle cerebral artery occlusion using Overhauser-enhanced magnetic resonance imaging. J Cereb Blood Flow Metab 29:1655-1664.

Yellon DM, Hausenloy DJ (2007) Myocardial reperfusion injury. N Engl J Med 357:1121-1135.

Yokoyama H, Ueda Y, Itoh O, Ikeda T, Noor JI, Ikenoue T (2004) EPR imaging to estimate the in vivo intracerebral reducing ability of mature rats after neonatal hypoxic-ischemic brain injury. Magn Reson Imaging 22:1305-1309. 